\title{
Capitated Payments to Primary Care Providers and the Delivery of Patient Education
}

\author{
William S. Pearson, PhD, MHA, Dana E. King, MD, MS, \\ and Chesley Richards, $M D, M P H$
}

Introduction: Patient education is a critical component of the patient-centered medical home and is a powerful and effective tool in chronic disease management. However, little is known about the effect of practice payment on rates of patient education during office encounters.

Methods: For this study we took data from the 2009 National Ambulatory Medical Care Survey. This was a cross-sectional analysis of patient visits to primary care providers to determine whether practice payment in the form of capitated payments is associated within patient education being included more frequently during office visits compared with other payment methods.

Results: In a sample size of 9863 visits in which capitation status was available and the provider was the patient's primary care provider, the weighted percentages of visits including patient education were measured as a percentages of education ( $95 \%$ confidence intervals): $<25 \%$ capitation, $42.7 \%(38.3-$ $47.3)$; $26 \%$ to $50 \%$ capitation, $37.6 \%(23.5-54.2)$; $51 \%$ to $75 \%$ capitation, $38.4 \%(28.1-49.8)$; $>75 \%$ capitation, $74.0 \%(52.2-88.1)$. In an adjusted logistic model controlling for new patients (yes/no), number of chronic conditions, number of medications managed, number of previous visits within the year, and age and sex of the patients, the odds of receiving education were reported as odds ratios (95\% confidence intervals): $<25 \%$ capitation, 1.00 (1.00-1.00); $26 \%$ to $50 \%$ capitation, $0.77(0.38-$ $1.58) ; 51 \%$ to $75 \%$ capitation, 0.81 (0.53-1.25); and $>75 \%$ capitation, 3.38 (1.23-9.30).

Conclusions: Patients are more likely to receive education if their primary care providers receive primarily capitated payment. This association is generally important for health policymakers constructing payment strategies for patient populations who would most benefit from interventions that incorporate or depend on patient education, such as populations requiring management of chronic diseases. (J Am Board Fam Med 2013;26:350-355.)

Keywords: Capitation Fee, Delivery of Health Care, Patient Education, Primary Health Care

Patient education is an important tool for physicians in preventing the deterioration of health due to illness and

This article was externally peer reviewed.

Submitted 13 November 2012; revised 10 January 2013; accepted 20 January 2013.

From the Office of Prevention through Healthcare, Centers for Disease Control and Prevention, Atlanta, GA (WSP, CR); the Department of Health Services, Policy and Management, University of South Carolina, Columbia (WSP); and the Department of Family Medicine, West Virginia University, Morgantown (DEK).

Funding: none.

Disclaimer: The views presented in this article are those of the authors and do not necessarily represent the views of the Centers for Disease Control and Prevention.

Conflict of interest: none declared.

Corresponding author: William S. Pearson, $\mathrm{PhD}$, Office of Prevention through Healthcare, Office of the Associate Director for Policy, Centers for Disease Control and Prevention, 1600 Clifton Road, Atlanta, GA 30333 (E-mail: Wpearson@cdc.gov). for promoting healthy lifestyle choices. ${ }^{1}$ Multiple studies have examined interventions involving patient education, including several emphasizing shared decision making. ${ }^{2}$ Benefits of greater patient education include improved use of value-based choices, improved patientpractitioner communication, increased involvement of the patient in decision making, and improved knowledge and realistic perception of outcomes. ${ }^{3}$ For example, a nurse-led intervention to educate patients regarding nonpharmacologic strategies to reduce gastroesophageal reflux demonstrated reduced patient symptoms ${ }^{4}$; similarly, a patient education program to promote exercise resulted in greater exercise tolerance and fewer costs for patients with chronic obstructive pulmonary disease. ${ }^{5}$

Numerous payment structures, including traditional fee for service, risk-adjusted pay for performance, capitation, and bundled payments, have 
been discussed by health services researchers with regard to their impact on the quality of care delivered to patients. ${ }^{6,7}$ Recent calls for increased quality of care and reduced cost of care at a population level have focused on the redesign of primary care. ${ }^{8}$ Because of the accelerating interest in payment reform and accountable care organizations, there has been renewed interest in the effect of population-based capitated payment on quality of care. ${ }^{9}$

Previous work has shown that there are differences in quality of primary care when comparing traditional fee-for-service payment arrangements to capitated payment systems. ${ }^{10}$ It is understood that capitated payment systems have significant effects on how physicians deliver care ${ }^{11}$ and how patients trust their physicians. ${ }^{12}$ However, the effect of capitated payment structures on the provision of patient education in particular has not been fully elucidated in the literature.

Therefore, we examined the association between the proportion of revenue received through capitation within a given practice and the proportion of patient visits indicating patient education was provided during the visit. We hypothesized that practices with higher levels of capitated payments would more often include patient education as part of a primary care outpatient visit.

\section{Methods}

We took the data for this study from the 2009 National Ambulatory Medical Care Survey, a nationally representative survey of office visits made to non-federally employed physicians in private offices and community health centers throughout the United States. The Centers for Disease Control and Prevention's National Center for Health Care Statistics conducts this survey annually, and it is exempt from approval by the Centers for Disease Control and Prevention's institutional review board.

The survey employs a multistage probability design that accounts for 3 stages of probability sampling - geographic primary sampling units, physician practices within the primary sampling units, and patient visits within the physician practices- to produce weighted national estimates of office visits. Office personnel use collection forms provided by the survey to obtain information about the visits, including patient demographics, medical conditions, treatments and medications ordered or pro- vided at the time of the visit, as well as other office-related information, during a 1-week sampling period. ${ }^{13}$

This study entailed a cross-sectional analysis of data collected from visits where the provider identified themselves as the patient's primary care provider. Among these visits, levels of capitation for the practice were collected via a variable within the dataset that featured 4 levels of capitation: $<25 \%, 26 \%$ to $50 \%, 51 \%$ to $75 \%$, and $>75 \%$. These levels of capitation were predetermined in the survey and a continuous measure of capitation was not available. Patient education was determined through a yes/no response to a question asking whether patient education was either provided or ordered at the time of the visit. Further information about the visit that was collected included the patient's age and sex, whether the patient had been seen before by the practice, the number of visits made by the patient to the practice within the past 12 months, the number of chronic conditions mentioned during the visit, the number of medications managed during the visit, and the expected source of payment for the visit. Ownership of the practice was a variable available within the dataset and included health maintenance organization (HMO) as an option. However, analysis of this variable found that $<2 \%$ of the total number of sampled visits met this definition. More than $75 \%$ of all sampled visits were made to a practice that was owned by a physician or physician group. Therefore, it was not possible to stratify ownership type by levels of revenue capitation because of the small sample size.

The total sample size used for analyses was 9863 patient visits. Initially, we used univariate descriptive analyses to demonstrate a general characterization of the sample. We then conducted bivariate analyses using $\chi^{2}$ tests to determine significant differences in patient education across the different levels of practice capitation. Finally, we used a logistic regression model to test differences among the different levels of practice capitation while taking into account other characteristics of the visits (mentioned earlier). All analyses were conducted using SUDAAN software (RTI International, Research Triangle Park, NC; available at http:// www.rti.org/sudaan/) to account for the complex sampling design of the survey and to produce national estimates. 
Table 1. Visit Characteristics $(n=9863)$ by Levels of Practice Capitation

\begin{tabular}{lcccc}
\hline & \multicolumn{4}{c}{ Levels of Capitated Payments } \\
\cline { 2 - 5 } Visit Characteristics & $<25 \%$ & $25-50 \%$ & $51-75 \%$ & $>75 \%$ \\
\hline Visits (n) & 8584 & 582 & 537 & 160 \\
Weighted n (in millions) & 347.1 & 15.2 & 19.4 & 7.1 \\
Mean age of patients, years & $42.6(39.6-45.5)$ & $39.0(26.6-51.4)$ & $31.1(9.5-52.6)$ & $40.0(24.0-55.9)$ \\
Female (\%) & $57.4(55.4-59.4)$ & $46.7(39.2-54.4)$ & $56.8(48.1-65.1)$ & $67.0(57.7-75.2)$ \\
Mean visits in past 12 months & $4.8(4.5-5.1)$ & $4.4(3.7-5.1)$ & $4.8(3.1-6.5)$ & $3.7(3.0-4.5)$ \\
Mean chronic illnesses managed & $1.4(1.2-1.5)$ & $1.4(0.8-2.0)$ & $1.2(0.1-2.3)$ & $1.7(0.9-2.4)$ \\
Mean medications managed & $2.9(2.6-3.2)$ & $2.7(2.0-3.3)$ & $2.5(1.2-3.9)$ & $2.8(2.0-3.6)$ \\
Patients seen before in the practice & $97.0(96.4-97.6)$ & $96.2(92.9-98.0)$ & $97.4(97.4-98.8)$ & $93.8(87.1-97.1)$ \\
$\quad$ Medicare/private insurance as expected & $83.0(78.8-86.5)$ & $83.2(67.9-92.0)$ & $58.7(32.6-86.7)$ & $79.4(56.0-90.2)$ \\
$\quad$ source of payment & & & & \\
\hline
\end{tabular}

Values are percentage estimates (95\% confidence intervals) unless otherwise indicated. Data are taken from the 2009 National Ambulatory Medical Care Survey.

\section{Results}

The sample used in these analyses represented an estimated total of nearly 390 million patient visits. The vast majority (347.1 million) were visits to practices that had a rate of capitated payment $<25 \%$, representing approximately $89 \%$ of the total sample. Visits to practices with capitation rates of $25 \%$ to $50 \%$ represented nearly $4 \%$ of the sample, visits to practices with capitation rates of $51 \%-$ $75 \%$ represented nearly $5 \%$ of the sample, and visits to practices with capitation rates of $>75 \%$ represented nearly $2 \%$ of the sample. Across the different levels of capitation there were no significant differences in the average age of patients seen, the percentage of female patients seen, the average number of chronic illnesses being managed, the average number of medications being managed, the percentage of visits by established patients, the average number of visits by the patients within the past 12 months, or the percentage of visits where the expected primary source of payment was either Medicare or private insurance (Table 1).

When we examined the percentage of visits where patient education was either ordered or delivered at the time of the visit, again, we found no significant differences across the different levels of capitation. We found that among visits to practices that received $>75 \%$ of their revenue from capitation, a larger proportion of visits had patient education involved compared with the proportion of visits to practices with lower levels of capitation. However, this proportion was not significantly higher (Table 2).
The relationship between patient education and levels of practice capitation was more fully elucidated using logistic regression models. In an unadjusted model where practices receiving less than $25 \%$ of their revenue from capitation was set as the reference, visits to practices with $>75 \%$ capitation were nearly 4 times as likely to have patient education associated with the visit (odds ratio, 3.81; 95\% confidence interval, 1.44-10.09). This effect was somewhat attenuated in a fully adjusted model controlling for patient age and sex, whether the patient was established in the practice, the number of visits made to the practice within the past 12 months by the patient, the number of chronic illnesses being managed, the number of medications being managed, and expected payment source for the visit. Even so, these visits were more than 3 times as likely (odds ratio 3.38; 95\% confidence interval, 1.23-9.30) to involve patient education (Table 3).

Table 2. Percentage Estimates of Visits With Patient Education ( $n=9863)$ by Level of Practice Capitation*

\begin{tabular}{lc}
\hline Level of Practice Capitation (\%) & $\begin{array}{c}\text { Visits With Patient } \\
\text { Education }\end{array}$ \\
\hline$<25$ & $42.7(38.3-47.3)$ \\
$25-50$ & $37.6(23.5-54.2)$ \\
$51-75$ & $38.4(28.1-49.8)$ \\
$>75$ & $74.0(52.2-88.1)$ \\
\hline
\end{tabular}

Values are percentage estimates (95\% confidence intervals). Data are taken from the 2009 National Ambulatory Medical Care Survey.

${ }^{*} P=.18, \chi^{2}$ test. 
Table 3. Logistic Regression Models Demonstrating the Likelihood of Patient Education by Level of Practice Capitation

\begin{tabular}{lccc}
\hline $\begin{array}{l}\text { Level of Practice } \\
\text { Capitation (\%) }\end{array}$ & $\beta$ & Odds Ratio & $\begin{array}{c}95 \% \text { Confidence } \\
\text { Interval }\end{array}$ \\
\hline $\begin{array}{l}\text { Unadjusted model } \\
>75\end{array}$ & & & \\
$50-75$ & 1.34 & 3.81 & $1.44-10.09$ \\
$25-50$ & -0.18 & 0.83 & $0.50-1.38$ \\
$<25$ & -0.21 & 0.81 & $0.40-1.62$ \\
Adjusted model & Reference & Reference & Reference \\
$>75$ & 1.22 & 3.38 & $1.23-9.30$ \\
$50-75$ & -0.21 & 0.81 & $0.53-1.25$ \\
$25-50$ & -0.26 & 0.77 & $0.38-1.58$ \\
$<25$ & Reference & Reference & Reference \\
\hline
\end{tabular}

Total visits, $\mathrm{n}=9863$. Data are taken from the 2009 National Ambulatory Medical Care Survey.

*Controlling for age and sex of patient, whether patient has been seen in practice before, number of visits to the practice in previous 12 months, number of chronic medical conditions, number of medications managed, expected payment source for the visit, and ownership of the practice.

\section{Discussion}

Our analyses suggest that capitated payments are associated with increased delivery of health education to patients in a primary care setting. There are a couple of possible explanations for this finding, the first being the focus on prevention as a cost reduction strategy in a managed care environment. It is possible that patient education is provided as a means of giving the patient more information on how to manage chronic conditions before they become acute and require more costly emergency care. Another possible explanation of this finding may be that there is a reduced focus on patient volume in a capitated environment compared with a fee-for-service environment. However, it is not possible to determine the plausibility of either of these explanations because of the lack of more detailed data on practice characteristics such as ownership of the practice. However, our data does provide a preliminary snapshot of national differences in the provision of patient education, which is a proven tool in the management of chronic health conditions, by levels of revenue received through capitation within a practice.

As health care reform unfolds and discussions on compensation strategies evolve, one method of reimbursement that is beginning to gain greater traction is capitated payments. Researchers have hy- pothesized that these capitated payments not only will reduce overall treatment costs in the long run, but that they are an incentive to provide care that will help reduce many unnecessary treatments in the short term and replace these treatments with more long-term solutions to good health, such as education about living a healthy lifestyle and selfmanagement of chronic disease.

Previous work has examined the effects of capitated payments on the delivery of services in a primary care setting. One such study examined the relationship between the level of managed care penetration within a practice and treatment behavior by physicians across patients. ${ }^{14}$ Those authors found that in practices where there are varied payment sources, duration of the visit across patients, all with varied payment sources, remains constant as managed care penetration increases within the practice. However, in these same practices, among patients who fall under a capitated payment system, the number of medications prescribed during the visit drops. The authors concluded that changes in payment methods can change physician behaviors and that physicians adopt a treatment style that delivers similar treatment intensity to all patients regardless of payment source.

Another similar study by Landon and colleagues $^{15}$ found that physicians who were in a highly capitated environment had lower total costs for patients covered under capitation and the intensity of care for these patients also was lower. These authors suggest that capitation is a strong incentive against providing additional services. However, there was no mention of the provision of health education, which could be a factor in controlling costly chronic conditions.

Recent work has examined multiple variations in primary care reimbursement, including full capitation as well as pay-for-performance models. However, critics of these models have pointed out that many of the proposed payment mechanisms fall short of fairly compensating primary care physicians for the complex management of multiple chronic diseases. Bereneson and Rich ${ }^{16}$ noted that as the public health care system begins to rely more heavily on primary care, extensive changes to the way primary care physicians are reimbursed for their work are necessary. Berenson and Rich ${ }^{17}$ go on to propose numerous payment plans combining traditional fee-for-service reimbursement with perpatient, per-month capitation payments that sup- 
port the patient centered medical home concept, which is argued to be the lynchpin for a reformed health care system.

Gorrol and colleagues ${ }^{18}$ have proposed a payment system that goes beyond a basic bundled payment method and extends reimbursement in a risk-adjusted manner to better compensate primary care physicians who take care of patients with multiple chronic conditions requiring complex care. More recently, Ash and Ellis ${ }^{19}$ used large claims databases to propose a payment model called the "primary care activity level model," which is a further extension of a risk-adjusted capitated payment and takes into account patients' socioeconomic status to predict outcomes. In this model, primary care physicians were reimbursed more accurately for the care that they provided by taking into account a better picture of the whole patient and the desired outcomes for each patient, rather than simply adjusting patient pools for risk.

\section{Limitations}

The findings of this study need to be considered in light of a number of limitations. First, this is a cross-sectional study; therefore, we could establish only a limited temporal relationship between capitation and the provision of health education. A stronger relationship between the two variables could be established through a longitudinal design examining capitated payments and delivery of health education over time. These data demonstrate a statistical association at only one point in time.

Second, we could not determine from these data whether each discrete visit was specifically reimbursed through a capitated payment. We could determine only the percentage of all payments received in the form of capitation for the entire office. Furthermore, many visits are reimbursed by multiple payers at different proportions of payment. A more granular data source that shows not only the expected payment source for a visit but also whether the visits were reimbursed from a larger capitation scheme would allow for a better understanding.

Third, it was not possible to determine the exact structure of the practice in terms of its ownership and governance. An ownership variable was available in the dataset that defined ownership as being privately owned or being owned by an HMO, a community health center, an academic medical center, or a hospital or other health care organiza- tion. During analyses, however, it was determined that $<2 \%$ of all practices in the available data were owned by an HMO. Therefore, it was not possible to stratify these practices by levels of capitated revenue because of small sample sizes. Since a small number of practices were owned by an HMO, it is believed that this would not have had a significant effect on the adjusted regression outcomes. Furthermore, practice ownership was highly correlated with the percentage of revenue that was received through capitation, which would have resulted in confounding.

Fourth, the number of medications being managed and the number of chronic medical conditions for each patient are crude measurements of visit acuity. It is possible that patients with lower numbers of medications and different types of chronic conditions may require a higher level of care that was not assessed in this data.

Fifth, the definition of levels of capitated revenue in the dataset did not allow for a threshold effect to be tested. The levels were set at $<25 \%$, $25 \%$ to $50 \%, 51 \%$ to $75 \%$, and $>75 \%$. Future data collection could ask for a more granular estimate for revenue received through capitation, which would allow for better explanation of changes in practice behavior.

Finally, the sample size for visits to practices with higher levels of capitated payments was relatively small compared with the number of visits to practices with lower levels of capitated payments. This situation is suspect for the nonsignificant findings in the bivariate analyses. Among visits to practices with higher levels of capitated payments, the percentage of patients receiving health education was considerably higher. However, those results were not significantly different compared with the other levels of capitated payments because the smaller numbers of visits produced larger confidence intervals for the percentage estimates. We suspect that a larger sample size would have produced significant outcomes in the bivariate analyses. Even so, multivariate analyses demonstrated the significant relationship between increased capitation and the delivery of health education.

\section{Conclusions}

Despite these limitations, this study has provided a preliminary picture of capitation and the delivery of 
health education in a primary care setting using recent nationally representative data. These findings speak to the current national discussion of how best to reduce rising health care costs while at the same time retaining or even increasing the quality of care that is delivered.

New systems of health care delivery that focus on population health are now developing, and primary care physicians along with patient-centered medical homes are at the root of such systems. ${ }^{20}$ One of the foundations of the new model of care is the accountable care organization, which relies on primary care practitioners to decrease health care costs within a population, while at the same time increasing the overall health of that population. However, debates over the most efficient reimbursement methods for this type of system seem to center on pay-for-performance measures that target defined patient populations rather than communities at large. ${ }^{21}$ This model may work well with specific populations, but it may not benefit patients outside of the target diagnoses. Therefore, discussions of new health care reimbursement models could consider patient education an important factor in promoting population health. Finding innovative reimbursement methods that reduce cost and increase quality for all patients is necessary to achieving a better performing US health care system.

\section{References}

1. Institute of Medicine. Crossing the quality chasm: a new health system for the 21 st century. Available from: http://www.iom.edu/Reports/2001/Crossingthe-Quality-Chasm-A-New-Health-System-for-the21st-Century.aspx. Accessed May 11, 2012.

2. Legarge F, Turcotte S, Stacey D, et al. Patients' perceptions of sharing in decisions: a systematic review of interventions to enhance shared decision making in routine clinical practice. Patient 2012;5:1-19.

3. Stacey D, Bennett CL, Barry MJ, et al. Decisions aids for people facing health treatment or screen decisions. Cochrane Database Syst Rev 2011;(10): CD001431.

4. Dibley LB, Norton C, Jones R. Non-pharmacological intervention for gastro-oesophageal reflux disease in primary care. Br J Gen Pract 2010;60:e459-65.

5. Ninot G, Moullec G, Picot MC, et al. Cost-saving effect of supervised exercise associated to COPD self-management education program. Respir Med 2011;105:377-85.

6. Pine M, Fry DE, Jones BL, et al. Controlling costs without compromising quality: paying hospitals for total knee replacement. Med Care 2010;48:862-8.

7. Bitton A, Martin C, Landon BE. A nationwide survey of patient centered medical home demonstration projects. J Gen Intern Med 2010;25:584-92.

8. Berwick DM, Nolan TW, Whittington J. The triple aim: care, health and cost. Health Aff (Millwood) 2008;27:759-69.

9. Mechanic RE, Santos P, Landon BE, et al. Medical group responses to global payment: early lessons from the 'Alternate Quality Contract' in Massachusetts. Health Aff (Millwood) 2011;30:1734-42.

10. Safran DG, Tarlov AR, Rogers WH. Primary care performance in fee-for-service and prepaid health care systems. JAMA 1994;271:1579-86.

11. Goodson JD, Bierman AS, Fein O, et al. The future of capitation: the physician role in managing change in practice. J Gen Intern Med 2001;16:250-6.

12. Kao AC, Green DC, Zaslavsky Am, et al. The relationship between method of physician payment and patient trust. JAMA 1998;280:1708-14.

13. Centers for Disease Control and Prevention (CDC). Ambulatory health care data. About the ambulatory health care surveys. Available from: http://www.cdc. gov/nchs/ahcd/about_ahcd.htm. Accessed May 17, 2013.

14. Glied S, Zivin JG. How do doctors behave when some (but not all) of their patients are in managed care? J Health Econ 2002;21:337-53.

15. Landon BE, Reschovsky JD, O'Malley AJ, Pham $\mathrm{HH}$, Hadley J. The relationship between physician compensation strategies and the intensity of care delivered to Medicare beneficiaries. Health Serv Res 2011;46:1863-82.

16. Berenson RA, Rich EC. U.S. approaches to physician payment: the deconstruction of primary care. J Gen Intern Med 2010;25:613-8.

17. Berenson RA, Rich EC. How to buy a medical home? Policy options and practical questions. J Gen Intern Med 2010;25:619-24.

18. Goroll AH, Berenson RA, Schoenbaum SC, Gardner LB. Fundamental reform of payment for adult primary care: comprehensive payment for comprehensive care. J Gen Intern Med 2007;22:410-5.

19. Ash AS, Ellis RP. Risk-adjusted payment and performance assessment for primary care. Med Care 2012; 8:643-53.

20. Sweeney SA, Bazemore A, Phillips RL, Etz RS, Stange KC. A reemerging political space for linking person and community through primary health care. Am J Public Health 2012;S3:S336-41.

21. Gourevitch MN, Cannell T, Boufford JI, Summers C. The Challenge of Attribution: Responsibility for Population Health in the Context of Accountable Care. American Journal of Public Health 2012;S3: S322-S324. 